\title{
StoryVR: A Virtual Reality App for Enhancing Reading
}

\author{
Federico Pianzola ${ }^{1,2}$ and Luca Deriu ${ }^{3}$ \\ 1 University of Milano-Bicocca, Milan, Italy \\ 2 Sogang University, Seoul, South Korea \\ federico.pianzola@unimib.it \\ 3 PlaySys, Milan, Italy
}

\begin{abstract}
We present a virtual reality app specifically designed for reading/listening to short stories and poems. Empirical studies with VR narrative experiences have proven that the process of embodied simulation enhanced by the VR medium increases users' absorption and engagement. Accordingly, this solution can be effectively used to promote reading and increase motivation for learning. We discuss the design choices adopted to facilitate its widespread adoption and maximise readers' engagement with stories.
\end{abstract}

Keywords: virtual reality, reading, literature, audiobook, narrative absorption

\section{Introduction}

Digital technologies have been exploited in different ways in relation to literature: by literary artists to create new text forms, by digital humanists for curation and criticism, but no one focused on designing literary texts in virtual reality (VR) to enhance traditional reading experiences. Story VR is the first VR app specifically designed to make literature more appealing by enhancing the level of immersion/absorption experienced while listening to stories. In recent years a few solution for reading in VR have been released [1,3,4], including an official app by one of the leading distributors of VR headsets, the HTC Vivepaper [2], which however is not maintained anymore. All these apps transpose the two-dimensional design of the page into a $3 \mathrm{D}$ space replicating the interactive affordance of paper books, that is requiring the reader to turn pages in order to continue the story. With Story VR we decided to take advantage of the increasing popularity of different form of experiencing literature - audiobooks - freeing the user from gestures related to another media and enabling developers to focus on the multimodal integration of audio and visual elements in order to enhance the engagement with the story.

\section{Virtual Reality and Electronic literature}

Virtual reality is a medium used to create experiences that are perceived as highly immersive [6]. VR environments have several positive effects when used 
as the context in which to perform ordinary activities, e.g. attention and sense of presence are increased [5], and emotional arousal is achieved [10,26]. However, VR has never been exploited to actively promote reading, an activity that could certainly benefit from increased immersion, attention and emotional engagement. Other kinds of digital technology have been used to create new literary formats, which are known as electronic literature: hypertexts, multimodal novels, interactive fiction, GPS location-specific narratives, installations in cave automatic virtual environments, etc. [14, 22, 11]. However, the majority of this kind of works is experimental and cannot be easily accessed by readers. On the contrary, we designed and developed a VR app intended for a widespread standalone VR Head Mounted Display (HMD) - Oculus Go - and with scalable content, since librarians and users are able to upload their own texts and audiobooks.

\section{$3 \quad$ Background research}

The Story VR experience has been designed on the basis of previous theoretical and empirical research on narrative absorption [13], situated reading [16], and presence in VR [17]. The theoretical assumption at the basis of StoryVR is that the level of immersion perceived when reading or listening to a story can be enhanced through environmental propping [16], that is the process for which features of the reading environment can foster readers' transportation into the story world, and empathy, eliciting a higher aesthetic pleasure. This hypothesis has been experimentally confirmed, showing that the perceived shift of embodiment from the actual world to the VR space facilitates a further shift of perceived embodiment into the story world [24]. The same study also found that VR can effectively be used to promote reading, since people who read in VR were more willing to continue reading. A similar research showed that when the VR experience is designed to let users embody the audience of a historical event, they feel a stronger sense of presence and are more willing to later seek more information about the event to which they participated [28]. However, although there is a widespread enthusiasm about the use of VR in education [29],VR does not seem to autonomously encourage learning in all fields: application to mathematics and science learning proved to be controversial $[18,19]$ if not supported by complementary learning strategies [23]. This process is in line with what predicted by cognitive theory of multimedia learning [8]. Overall, VR works well as a tool to enhance motivation and enthusiasm towards a topic, especially with narrative experiences that enable embodied simulation.

\section{Design and implementation}

The app is specifically designed for reading/listening to stories, creating distractionfree moments dedicated to this activity. For this reason, the user experience and interface have been designed with purpose-driven limitations. It is a seated experience with movements limited to 3-degrees-of-freedom, no interaction with 
the physical environment is possible, and the user interface (UI) is intuitive and requires only a few clicks to start reading/listening to the story.

\subsection{User Interface}

The UI is a non-diegetic panel in which users can choose an environment and a text/audiobook from two menus (Fig. 1). The panel with the two menus can be accessed by clicking on a 3D object floating in the scene (spatial interface), a closed old book that opens and reveals the panel. Users can pause the audiobook, change background scene and activate/deactivate the audio reverb (see below). By clicking again on the $3 \mathrm{D}$ book the panel disappears.

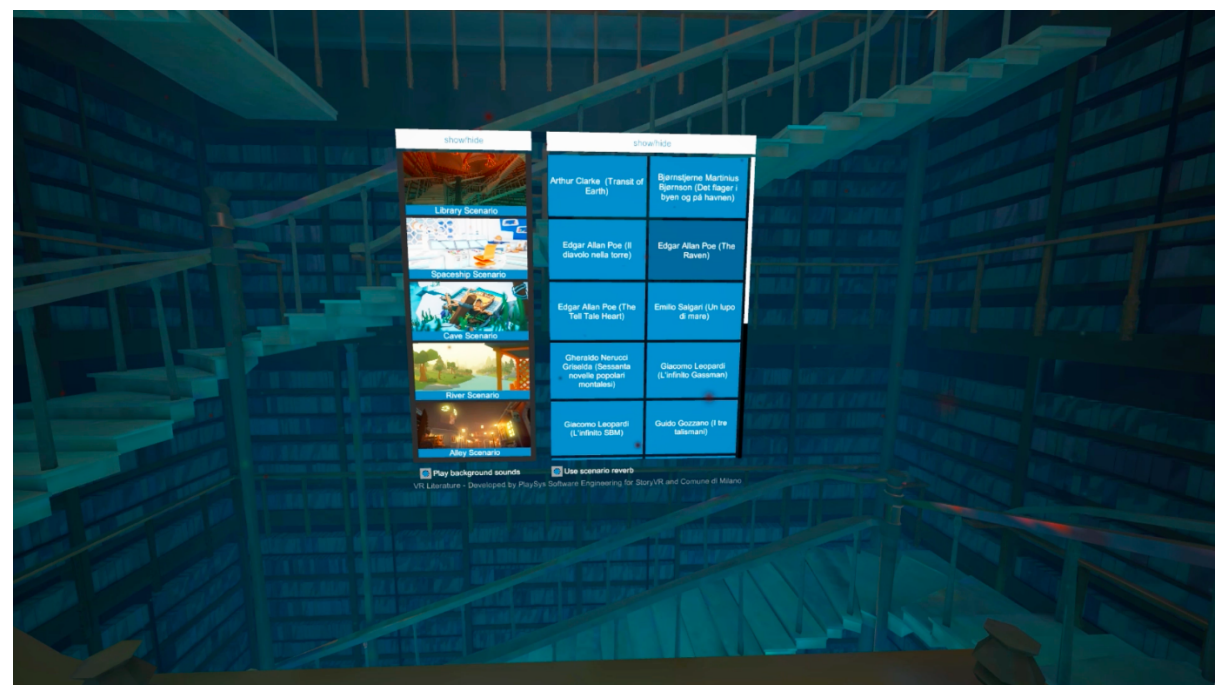

Fig. 1. Home scene with user interface for the selection of story and environment.

\subsection{Graphics}

A series of environments have been designed and manually modelled in 3D with the aim of creating an atmosphere that can help to focus on the reading experience and increase the absorption in the story by matching the environment with the story's theme or atmosphere. The home environment is a mysterious wooden library, in which users are surrounded by books. Here they can select a story or poem and one of four different environments (Fig. 2), designed to offer visual and audio stimuli that will be "peripherally perceived" during reading/listening, thus supporting imagery and transportation into the story world [16]. The scenes do not represent the story content, therefore we believe they will not be an impediment for imagination, since users will still be able to picture 
as they want the situation evoked by the story. On the contrary, having a visual prompt can help people who do not have a high mental imagery ability [20,12]. A $19^{\text {th }}$ century foggy alley at night is suitable for horror and mystery stories; a spacecraft, for science fiction; an underwater cave, for adventure and pirates stories. The fourth environment is a hut by the river, which is more neutral and has been conceived - based on users' feedback - as a relaxing place suited for a variety of stories (Fig. 3). The environments have been created with a low-poly graphic style, which has been chosen to make the experience pleasant for both children and adults, while keeping the graphic computing power required to a minimum, so that the app works smoothly even in low-specifications HMDs like the Oculus Go, which are more economically affordable for schools.

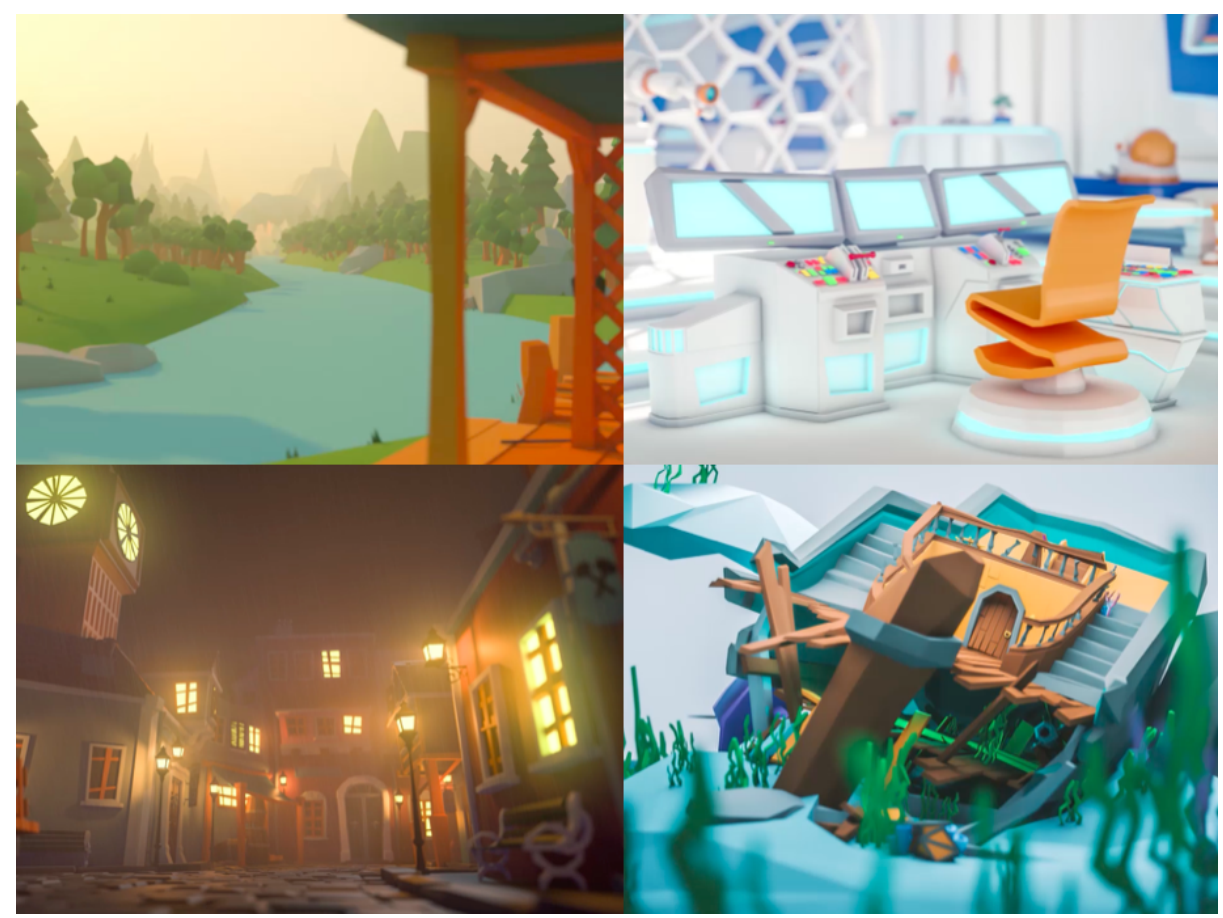

Fig. 2. Details of the four animated scenes that users can choose as environment while listening to audiobooks.

\subsection{Sounds}

Ambient sounds have been carefully selected and mixed to increase immersion. Every environment has its own specific background sounds and audio reverb zone that simulates the different spatial reflection of sound in each environment. 


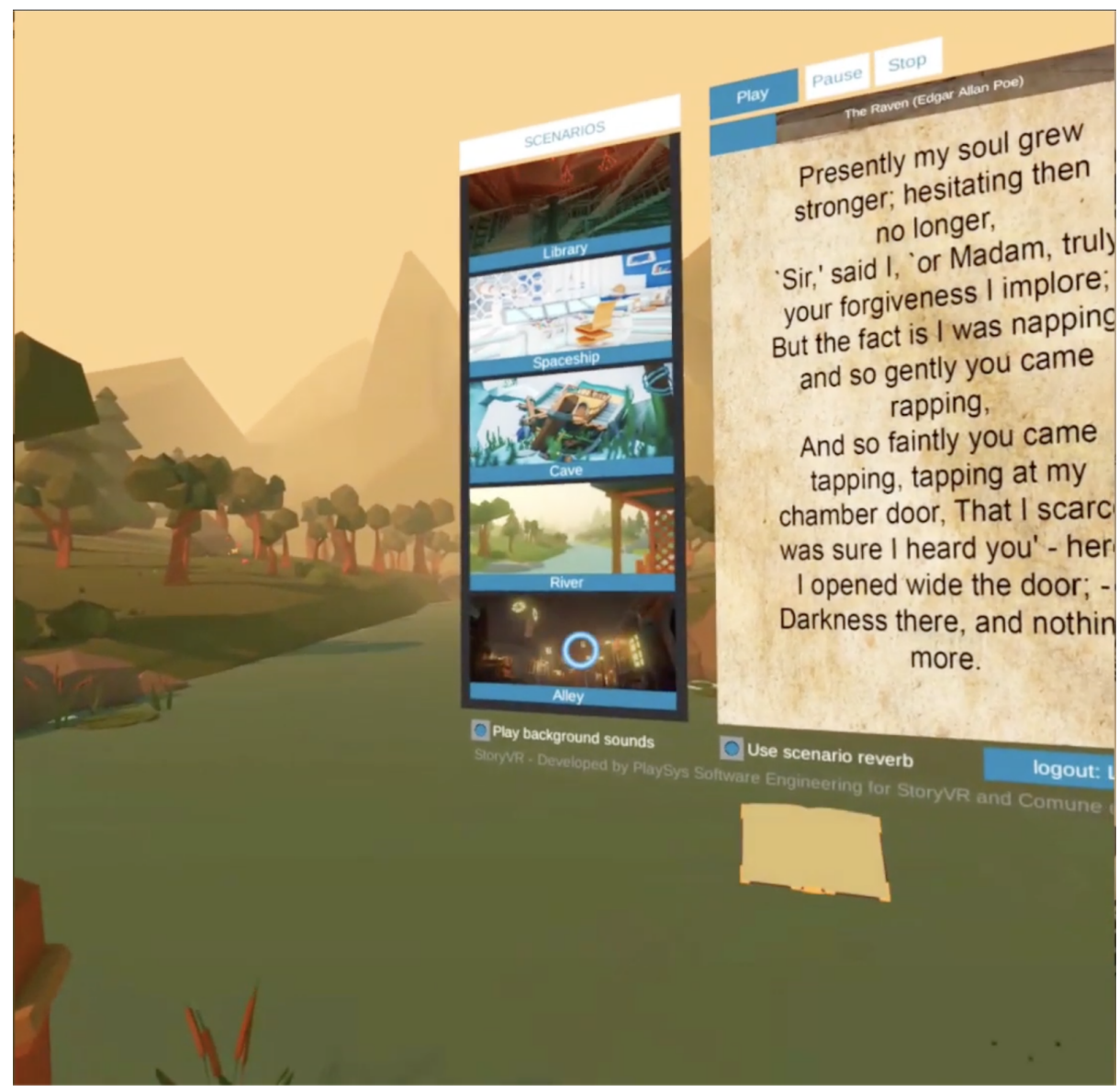

Fig. 3. Relaxing environment with the menu panel and text visible.

Moreover, we considered that when we read engaging narratives there is an unconscious narrowing of the attentional focus to the story, which consequently limits our epistemic awareness [27], that is the reduction of sensitivity to external stimuli. However, in VR the ambient sounds are perceived as part of the mediated experience and it is difficult for the user to "filter off" the background noise. Therefore, to simulate the perceptive attentional focus we used a script that progressively reduces the volume of the ambient sound when the audio of the story start or the text is displayed. This feature facilitates immersion into the story world.

\subsection{Virtual agent as narrator}

We are currently developing a virtual agent [25] that can function as a narrator. Having an agent that embodies the narrating voice in the VR environment will 
likely increase the sense of presence in the VR environment [15]. The goal is to reduce users' mind wandering by helping them keeping the attention on the story $[30,9]$. The virtual agent is programmed to have casual eye-contact with the user and the movement of the lips is synchronised with the audio of the selected story. Mouth shapes that create lip-syncing are based on phonemes and various sets of phonemes are available so that different languages can be simulated. Facial expressions and gestures are automatically synced to the speech to give appropriate emphasis to the story and create a more positive effect on users [21]. The implementation of this solution will increase the verisimilitude of the sense of co-presence of user and virtual agent and the feeling of a positive experience [7]. Previous research showed that, in a narrative context, embodied simulation of the audience is more effective than first-person embodiment of the protagonist for increasing sense of presence, engagement, and motivation to learn [28].

\subsection{User tracking}

For research purposes, we included in the app analytics tools to track the users' choices of scenes and stories. Data collected regard the amount of stories read/listened, the rate of abandonment, and the session time. This information can be used to understand reading preferences but also to investigate more in depth the relationship between reading and environmental stimuli. Used in combination with more sophisticated HMDs - e.g. with eye-tracking or EEG sensors - StoryVR can be used for neuropsychological research on reading. The advantage will be a greater control on environmental variables during experiments compared to laboratory settings, which do not usually offer a relaxed environment encouraging reading for enjoyment.

\section{Conclusion}

In the context of a no-profit project promoting reading, the Story VR app is first being distributed in public libraries together with HMDs, where specific spaces ("VR corners") are designed to host this new service. StoryVR is being used to attract young people to the library and to collect data about library users' preferences in matching environments and stories. In order to encourage the lifelong improvement of reading skills, using tasks that focus only on the development of reading comprehension is a solution with major shortcomings. In this respect, helping people to become passionate and motivated readers is of tantamount importance to sustain their future autonomous reading activities. By leveraging an attractive technology like virtual reality, whose learning and entertainment potential based on embodied simulation has been widely proved, the StoryVR app can be used in educational context to engage students, offering a new point of access to literary cultural heritage. We are currently doing experiments with quantitative and qualitative methods in Europe and South Korea, investigating how the environment influences the reading experience and whether cultural differences influence narrative absorption and the acceptance of the hybridisation 
of literature with VR technology. Users who tried the StoryVR app so far reported it to create both an "intimate" and "cultural" experience. Overall, the app proved to have a great potential for the promotion of reading and could be used for educational purposes as well as for leisure. Future improvements will be focused on adding sound effects and animations based on the progression of the story, in order to increase narrative absorption even further.

\section{References}

1. Chimera reader (2016). URL https://www.oculus.com/experiences/gear-vr/ $901261583298725 /$ ?locale $=$ en_GB

2. Vivepaper (2016). URL http://www.vivepaper.com/en.html

3. immersionVR reader (2017). URL https://www.oculus.com/experiences/go/ 1397503436990351/

4. VirtualBook Viewer (2018). URL https://www.oculus.com/experiences/go/ 2341468065880359/

5. Baños, R., Botella, C., Alcañiz, M., Liaño, V., Guerrero, B., Rey, B.: Immersion and Emotion: Their Impact on the Sense of Presence. CyberPsychology \& Behavior 7(6), 734-741 (2004). DOI 10.1089/cpb.2004.7.734

6. Bailenson, J.N.: Experience on Demand. What Virtual Reality is, How it works, and What it can do. Norton \& Company, New York and London (2018)

7. Bailenson, J.N., Beall, A.C., Loomis, J., Blascovich, J., Turk, M.: Transformed Social Interaction, Augmented Gaze, and Social Influence in Immersive Virtual Environments. Human Communication Research 31(4), 511-537 (2005). DOI 10.1111/j.1468-2958.2005.tb00881.x

8. Colvin Clark, R., Mayer, R.E.: e-Learning and the Science of Instruction : Proven Guidelines for Consumers and Designers of Multimedia Learning, third edn. Pfeiffer, San Francisco (2011)

9. Corballis, M.C.: The Wandering Mind: What the Brain Does When You're Not Looking. University of Chicago Press, Chicago (2015)

10. Diemer, J., Alpers, G.W., Peperkorn, H.M., Shiban, Y., Mühlberger, A.: The impact of perception and presence on emotional reactions: a review of research in virtual reality. Frontiers in Psychology 6(26) (2015). DOI 10.3389/fpsyg.2015.00026

11. ELMCIP: Electronic Literature Knowledge Base. URL https://elmcip.net/ knowledgebase

12. Green, M.C., Kass, S., Carrey, J., Herzig, B., Feeney, R., Sabini, J.: Transportation Across Media: Repeated Exposure to Print and Film. Media Psychology 11(4), 512-539 (2008). DOI 10.1080/15213260802492000

13. Hakemulder, F., Kuijpers, M.M., Tan, E.S., Bálint, K., Doicaru, M.M. (eds.): Narrative Absorption. John Benjamins, Amsterdam/Philadelphia (2017)

14. Hayles, N.K.: Electronic literature : New Horizons for the Literary. University of Notre Dame Press, Notre Dame (2008)

15. Kuzmičová, A.: Literary Narrative and Mental Imagery: A View from Embodied Cognition. Style 48(3,), 275-293 (2014)

16. Kuzmičová, A.: Does it Matter Where You Read? Situating Narrative in Physical Environment. Communication Theory 26(3), 290-308 (2016). DOI 10.1111/comt. 12084

17. Lombard, M., Biocca, F., Freeman, J., Ijsselsteijn, W., Schaevitz, R.J. (eds.): Immersed in Media: Telepresence Theory, Measurement \& Technology. Springer, Cham (2015). OCLC: 907095808 
18. Madden, J., Pandita, S., Schuldt, J.P., Kim, B., S. Won, A., Holmes, N.G.: Ready student one: Exploring the predictors of student learning in virtual reality. PLOS ONE 15(3), e0229,788 (2020). DOI 10.1371/journal.pone.0229788

19. Moreno, R., Mayer, R.E.: Learning science in virtual reality multimedia environments: Role of methods and media. Journal of Educational Psychology 94(3), 598-610 (2002). DOI 10.1037/0022-0663.94.3.598

20. Neumann, D., Moffitt, R.: Affective and Attentional States When Running in a Virtual Reality Environment. Sports 6(3), 71 (2018). DOI 10.3390/sports6030071

21. Oh, S.Y., Bailenson, J., Krämer, N., Li, B.: Let the Avatar Brighten Your Smile: Effects of Enhancing Facial Expressions in Virtual Environments. PLOS ONE 11(9), e0161,794 (2016). DOI 10.1371/journal.pone.0161794

22. Organization, E.L.: Electronic Literature Directory. URL http://directory. eliterature.org/

23. Parong, J., Mayer, R.E.: Learning science in immersive virtual reality. Journal of Educational Psychology 110(6), 785-797 (2018). DOI 10.1037/edu0000241

24. Pianzola, F., Bálint, K., Weller, J.: Virtual Reality as a Tool for Promoting Reading via Enhanced Narrative Absorption and Empathy. Scientific Study of Literature $\mathbf{9}(2)(2020)$

25. Qu, C.: Talking with a Virtual Human: Controlling the Human Experience and Behavior in a Virtual Conversation. Ph.D. thesis, TU Delft, Delft (2014). URL http://resolver.tudelft.nl/uuid:b4f77e2f-b6b8-493f-bc6e-e536dada300e

26. Riva, G., Mantovani, F., Capideville, C.S., Preziosa, A., Morganti, F., Villani, D., Gaggioli, A., Botella, C., Alcañiz, M.: Affective Interactions Using Virtual Reality: The Link between Presence and Emotions. CyberPsychology \& Behavior 10(1), 45-56 (2007). DOI 10.1089/cpb.2006.9993

27. Schwitzgebel, E.: Do You Have Constant Tactile Experience of Your Feet in Your Shoes? Or Is Experience Limited to What's in Attention? Journal of Consciousness Studies 14(3), 5-35 (2007)

28. Slater, M., Navarro, X., Valenzuela, J., Oliva, R., Beacco, A., Thorn, J., Watson, Z.: Virtually Being Lenin Enhances Presence and Engagement in a Scene From the Russian Revolution. Frontiers in Robotics and AI 5, 91 (2018). DOI 10.3389/ frobt.2018.00091

29. Slater, M., Sanchez-Vives, M.V.: Enhancing Our Lives with Immersive Virtual Reality. Frontiers in Robotics and AI 3 (2016). DOI 10.3389/frobt.2016.00074

30. Varao Sousa, T.L., Carriere, J.S.A., Smilek, D.: The way we encounter reading material influences how frequently we mind wander. Frontiers in Psychology 4 (2013). DOI 10.3389/fpsyg.2013.00892 